Title:

Author(s):

Submitted to:

\section{National Information Infrastructure} Applications

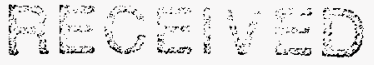

JUL 19199

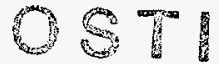

David Forslund, ACL

James George, ACL

Jonathan Greenfield, CIC-8

Mohamad ljadi, CIC-18

Niels Jensen, T-11

Patrick Kelly, ClC-3

Juhnyoung Lee, ACL

Allen McPherson, CIC-8

Richard Phillips, $A C L$

Stephen Tenbrink, CIC-5

DOE Office of Scientific and Technical

Information (OSTI)

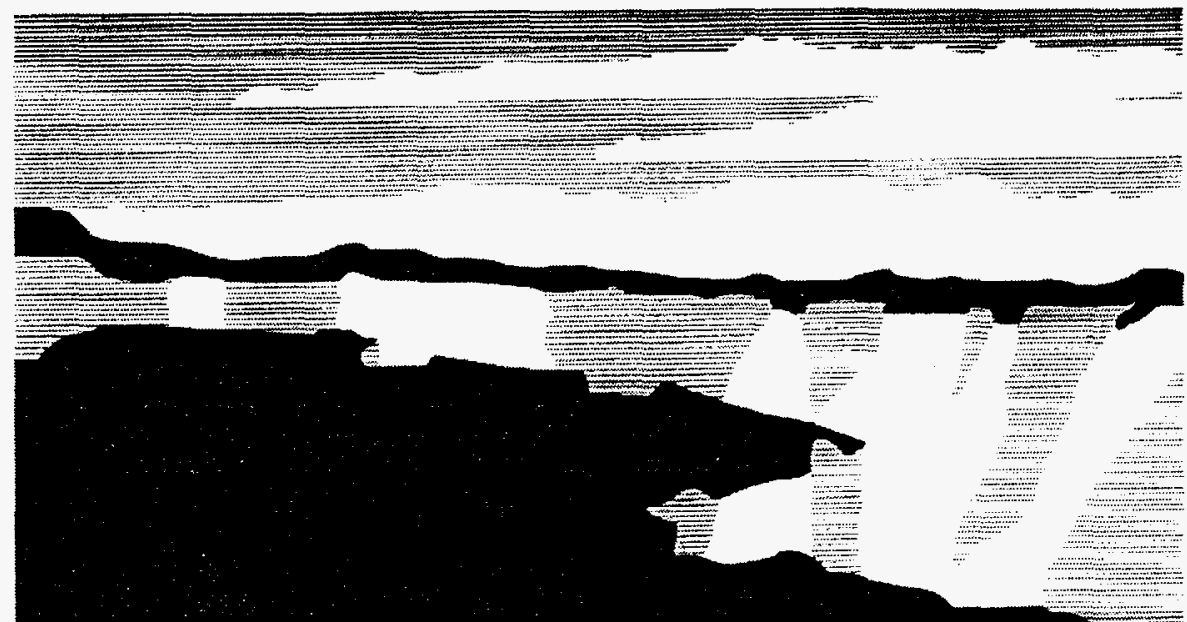

NATIONAL LABORATORY

Los Alamos National Laboratory, an attirmative actionequal opportunity employer, is operated by the University of Calfornia for the U.S. Department of Energy under contract W-7405-ENG-36. By acceptance of this article, the publisher recognizes that the U.S. Government retains a nonexclusive, royaltyfree license to publish or reproduce the published form of this contribution, or to allow others to do so, for U.S. Government purposes. The Los Alamos National Laboratory requests that the publisher identify this article as work performed under the auspice 3

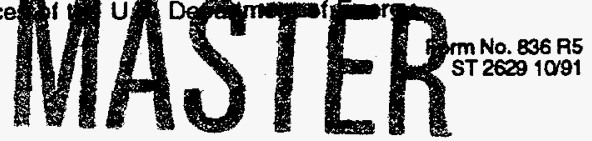




\section{DISCLAIMER}

Portions of this document may be illegible in electronic image products. Images are produced from the best available original document. 


\section{DISCLAIMER}

This report was prepared as an account of work sponsored by an agency of the United States Government. Neither the United States Government nor any agency thereof, nor any of their employees, makes any warranty, express or implied, or assumes any legal liability or responsibility for the accuracy, completeness, or usefulness of any information, apparatus, product, or process disclosed, or represents that its use would not infringe privately owned rights. Reference herein to any specific commercial product, process, or service by trade name, trademark, manufacturer, or otherwise does not necessarily constitute or imply its endorsement, recommendation, or favoring by the United States Government or any agency thereof. The views and opinions of authors expressed herein do not necessarily state or reflect those of the United States Government or any agency thereof. 


\title{
National Information Infrastructure Applications
}

David Forslund*, James George, Jonathan Greenfield, Mohamad Ijadi, Niels Jensen, Patrick Kelly, Juhnyoung Lee, Allen McPherson, Richard Phillips, and Stephen Tenbrink

\begin{abstract}
This is the final report of a two-year, Laboratory-Directed Research and Development (LDRD) project at the Los Alamos National Laboratory (LANL). This project sought to develop a telemedical application in which medical records are electronically searched and digital signatures of real CT scan data are indexed and used to characterize a range of diseases and are used to compare on-line medical data with archived clinical data rapidly. This system includes multimedia data management, interactive collaboration, data compression and transmission, remote data storage and retrieval, and automated data analysis integrated in a distributed application between Los Alamos and the National Jewish Hospital.
\end{abstract}

\section{Background and Research Objectives}

It is increasingly recognized that the management of information in an intelligent manner will be an increasingly strategic resource for the nation and for industry in the coming century. Competitiveness of the Nation will depend on it as will the competitiveness of the Laboratory. This project sought to develop a prototype of the National Information Infrastructure (NII) system and extend the knowledge gained from this into a variety of programs in the Laboratory. To achieve this goal, we set the following three objectives:

- To develop common information-enabling tools for advanced scientific research and its application to industry;

- To enhance the capabilities of important research programs at the Laboratory; and

- To define a new way of collaboration between computer science and scientific research and development.

The idea is to take real applications with wide impact and use them to drive the appropriate technology and infrastructure. The importance of this approach is widely recognized and indeed is an important component of almost every developing program in the Laboratory.

* Principal investigator, e-mail: dwf@lanl.gov 
In the first year of the project, we had several application areas that were driving the development of the infrastructure. In the second year, we focused on the most successful of these projects, telemedicine. Although this alone is of importance to the Laboratory, the model of telemedicine has wide applicability. Keeping track of a complex patient record over time and allowing one to quickly and easily navigate through it has many analogies throughout the Laboratory. A weapon system, for example, is much like a patient. It is born and endures over time, undergoing aging, perhaps damage. It must be assessed non-destructively to decide if an operation is required or desirable when problems are encountered, or treatment of some kind must be initiated. Finally, it must be determined if corrective action is necessary under any conditions. Thus we see immediate application of our system to the potential integration of the entire nuclear complex that deals with these problems. The effort to understand structure in images of importance to radiologists is also important in the materials area where one is trying to understand the causes of certain kinds of crystalline behavior or defects in solids. Being able to compare images intelligently across a wide variety of experiments is of great value and something that is not being handled today even though there has been a lot of money spent on new instruments that produce enormous amounts of image data.

\section{Importance to LANL's Science and Technology Base and National R\&D Needs}

This project supports Los Alamos core competencies in theory, modeling, and highperformance computing as well as bioscience and biotechnology. The project enhances the Laboratory's visibility in the area of information infrastructure and increases its ability to respond to initiatives in that area. As indicated above, the integration of complex information has applications throughout the entire DOE nuclear complex.

\section{Scientific Approach and Results to Date}

\section{Telemedicine}

The Sunrise Project is a coordinated effort to develop a nationally scalable, highperformance networked environment that can support a wide range of applications ranging from healthcare to materials and engineering data. The idea is to take a suite of applications to force the development of a common infrastructure that can be reused across applications and provide the capability of managing large amounts of information in an intelligent manner. This is done in a fully distributed, object-oriented system that can handle a variety of multimedia data types in a collaborative environment. The result is a fully integrated collaborative 
environment incorporating the most advanced concept extraction technologies in a ready-to-use application for the end user. TeleMed is the first complete application evolving from this software approach.

The specific TeleMed application grew out of a relationship with physicians at the National Jewish Center for Immunology and Respiratory Medicine (NJC) in Denver, Colorado. These physicians are experts in pulmonary diseases and radiology, helping patients combat the effects of tuberculosis and other lung diseases throughout the Nation. These individuals are an expensive and scarce resource who often travel around the country to share their expertise with other physicians.

To make the knowledge and experience at the National Jewish Center available to a wider audience, Los Alamos National Laboratory has developed a telemedicine system called TeleMed, which is based on a distributed national radiographic and patient record repository located anywhere in the country. Without leaving their offices, participating doctors can view radiographic data via a sophisticated multimedia interface. For example, a doctor can match a patient's radiographic information with the data in the repository, review treatment history and success, and then determine the best treatment. Furthermore, the features of TeleMed that make it attractive to clinicians and diagnosticians make it valuable for teaching and presentation as well. Thus, a resident can use TeleMed for self-training in diagnostic techniques and a physician can use it to explain to a patient the course of their illness. In fact, the data can be viewed simultaneously by users at two or more distant locations for consultation with specialists in different fields. This capability is of enormous value to a wide spectrum of healthcare providers.

Some of the capabilities of TeleMed can be illustrated by looking at a series of user interface components that are available to the user. The user begins a TeleMed session by selecting a database site from the interface shown in Figure 1. This sets in motion an Orbixbased transaction for vending all patient record objects from the selected site to the requesting client, shown listed in Figure 1 as Patient \#1, Patient \#2, etc. To understand the coordination of distributed object activities with user interface activities we can consult Figure 2, a graphical representation of TeleMed objects. In this diagram the arrows represent an inheritance relationship and the other lines represent a reference or containment. Textual data from the patient object (e.g., the patient's name) was retrieved and used to populate the patient list in Figure 1. A patient's treatment record appears by double-clicking on the patient's name in the interface in Figure 1. The user interface manifestation of the observation battery object in Figure 2 is called a graphical patient record (GPR) and is shown in Figure 3.

The GPR is an excellent example of Sunrise/TeleMed media-rich document and distributed object technology. The GPR is a virtual document, a patient record that is empty 
until it is dynamically populated by requests for distributed objects. The MedLib object in Figure 2 contains the information necessary for "harvesting" this data from all appropriate sites. Thus, laboratory reports may be retrieved from the National Institutes of Health near Washington, DC, while radiographic data may reside at the NJC in Denver. So, for example, when all patient data are retrieved, icons representing laboratory tests, radiographic studies, drug treatments, etc., are drawn on the GPR template. Each of these icons is mouse-sensitive and, when clicked, call up additional user interfaces and related data.

Before looking at these interfaces it will be helpful to know more about what goes on at the distributed objects level. In Figure 4 we show the relationships between the client process, TeleMed graphical user interface (GUT) and the two controlling objects, MedLibObj and MatchObj. Any of these three entities can reside at any location. In fact, the TeleMed GUI can communicate with any number of MedLibObj objects, which, in turn, can call upon the services of any number of MatchObj objects. Suppose the user clicks on a computerized tomography (CT) study icon in the GPR in Figure 3. This causes a request to be sent to the current MedLibObj to retrieve that patient's CT study from the corresponding persistent object store. That transaction causes the user interface shown in Figure 5 to appear. The image on the left of Figure 5 is a scout, so named because it was originally used by the CT technician as a guide in determining where to produce full transverse slice images of the patient. In this interface the scout is similarly used, but now as a guide for the physician in selecting slices to view from the database. To do this, the horizontal cursor is dragged up or down to the desired location and released. Here, slice number 23 was selected and is shown on the right.

We conclude our discussion of the TeleMed application by describing one of its most powerful features. This feature allows a user to perform a "query by example" search of an image database. Many technologies are represented in this feature: massively parallel computation servers, image analysis agents, and distributed object computing. To be specific, the MatchObj object shown in Figure 4 encapsulates the image analysis agent as a member function. For best performance MatchObj will typically reside on a massively parallel computer because the matching algorithm is inherently parallel. The signature database, which contains representative features of each image, usually resides on the same machine as MatchObj. Finally, the user invokes this entire matching operation simply by clicking the "Find Match" button in the upper right of Figure 5. The selected slice is used as the query image. The result of a matching operation is shown in Figure 6. In Figure 6 the upper left image is the same one the user specified as the example query image. The result of the match is summarized by the thumbnails in the lower scrolling window. Clicking in a selected thumbnail causes its full-size representation to appear in the upper right comparative inspection window. 
In the TeleMed application, we have developed a powerful environment for navigating through patient data both by browsing and by providing specialized search tools to show relationships between data that are not always obvious to the physician or the end user. The paradigm used here is very general, requiring only small modifications to be used on a wide range of disciplines. It is extensible in at least two ways. First, the ability to display virtual visual "metadata" whose spatial relationships conveys higher level relationships is a general capability with applicability far beyond medicine. Secondly, the idea of a "patient record" is directly applicable in engineering systems where one tracks a complex system over its entire life history from design and assembly to testing and evaluation at a later date after aging or potential damage. This can apply to a set of calculations or a set of experiments any of which have a complex and large amount of data to be investigated. This approach provides a vehicle for organizing the data and controlling access to it so the user isn't overloaded with information but is given enough of it to understand what is going on and to navigate through it.

Engineering systems frequently involve using much of the non-destructive testing techniques of value in the medical arena although frequently in different physical regimes. Systems can be compared with the imaging techniques used above to determine which ones may have had similar features or been through similar time histories. Computational results might be compared to earlier results where one recalls seeing something like these results, but doesn't know where.

We see an ever increasing need for proper organization and archiving of important information related to experimental as well as computational systems. TeleMed is the beginning of a new type of application in this area of information handling. In the healthcare field, TeleMed is one of the first implementations of important parts of what will be a computer-based, patient-record system. It is the first to provide transparent access to patient record components over a wide-area network, building the complete patient record from various partial records and displaying that in an integrated manner to the healthcare provider. It could provide an excellent platform with which to experiment with a variety of computer patient record concepts. This includes the newly added capability of telecollaboration between multiple physicians while viewing the identical data and commenting and documenting their observations. We envision extensions to add the ability to link multiple existing applications for processing the information as well as multiple user interfaces with differing domain specificities. The layer architecture we have adopted makes this possible with only modest amount of additional effort. 


\section{Networking}

At the early inception of the Sunrise Project, asynchronous transfer mode (ATM) was chosen as the network infrastructure for the high bandwidths and other requirements of Sunrise and the TeleMed Project that grew out of Sunrise. The reason for ATM's selection, in addition to higher bandwidths, was the promise of support for isochronous services, such as audio and video, for telecollaboration. Since ATM technology is still evolving, the approach taken was to set up an ATM Testbed at LANL to evaluate the different switches, interface cards, and other ATM capabilities. As of August, 1995, this Testbed had the following equipment:

- 1 Fore ASX-200 ATM switch with 4 OC-3 and 8 TAXI ports;

- 2 Cisco A100 ATM switches with 8 OC-3 ports each;

- 2 Fore SBA-200 TAXI interface cards for S-Bus;

- 2 Fore GIA-200 OC-3 interface cards for SGI's GIO bus;

- 1 Fore VME-200 TAXI interface card for SGI's Onyx bus (VME);

- 1 Efficient Network's OC-3 interface card for S-Bus;

- 1 Fore AVA-200 audio/video adapter for ATM networks with software for Sun/Sparc workstations; and

- 1 NetStar Gigarouter for routing between ATM, HIPPI, and FDDI networks.

\section{References}

Forslund, David, "TeleMed: A High Performance Distributed Application for the Real World," LLNL High Performance Distributed Computing Conference, LANL Report LA-UR \#95-1833 (1995).

Kelly, P.M.and Cannon, T.M., "CANDID: Comparison Algorithm for Navigating Digital Image Databases," in Proceedings of the 7th International Working Conference on Scientific and Statistical Database Management, LANL Report LA-UR \#94-0721 (1994).

Kelly, P.M.and Cannon, T.M., "Experience with CANDID: Comparison Algorithm for Navigating Digital Image Databases," SPIE, 2368, LANL Report LA-UR \#94-3086 (1994).

Kelly, Patrick; Cannon, Mike; and Hush, D.R., "Query by Image Example: the CANDID Approach," SPIE, 2420, LANL Report LA-UR \#95-374 (1995).

Forslund, David, "Advanced Distributed Multimedia Data Analysis and Display Technology: The Sunrise Project and its Application to Healthcare and Manufacturing," NCMS Technical Conference 4/95, LANL Report LA-UR \#95-1832 (1995).

Phillips, Richard, "Networkbased Distributed, Media-Rich Computing and Information Environment," Leeds Conference, LANL Report LA-UR \#94-3835 (1994). 


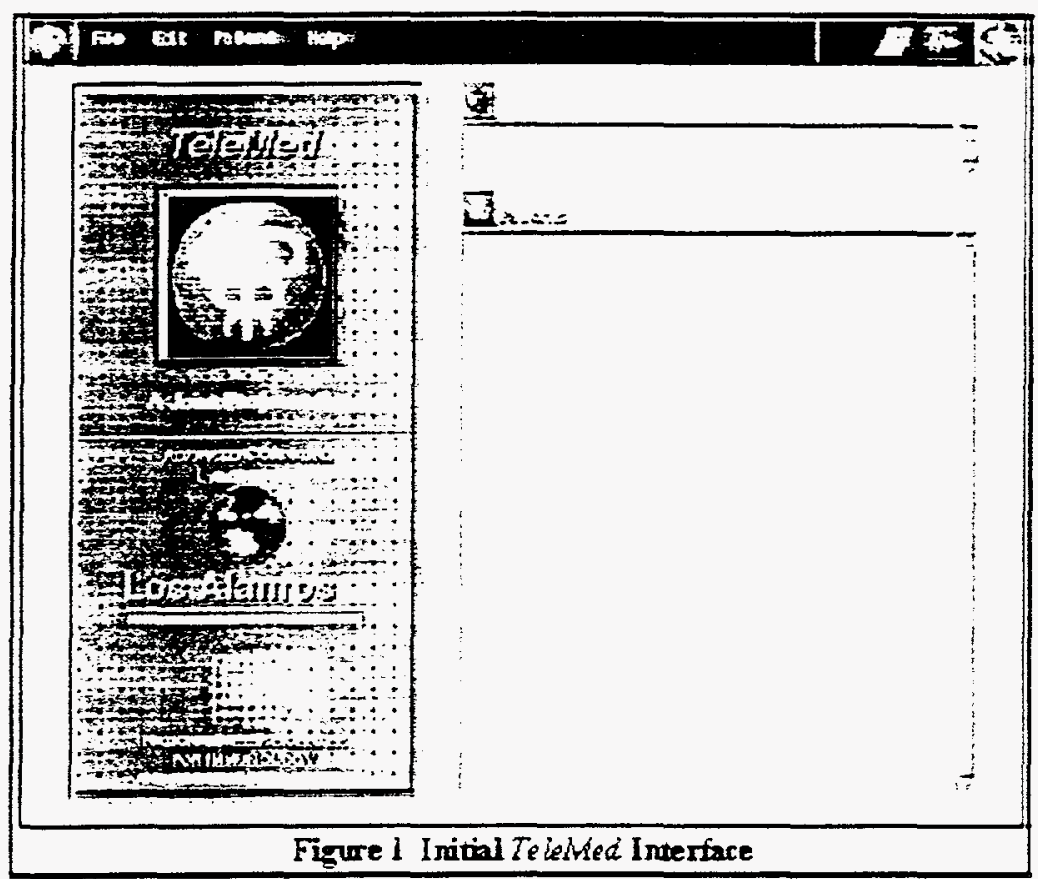

Figure 1. Initial TeleMed interface.

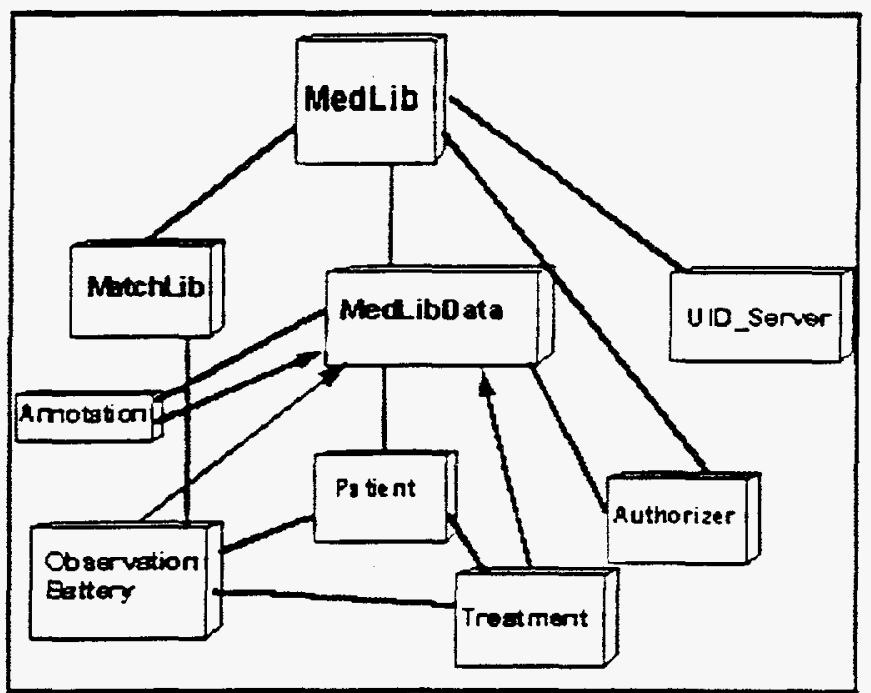

Figure 2. TeleMed objects. 


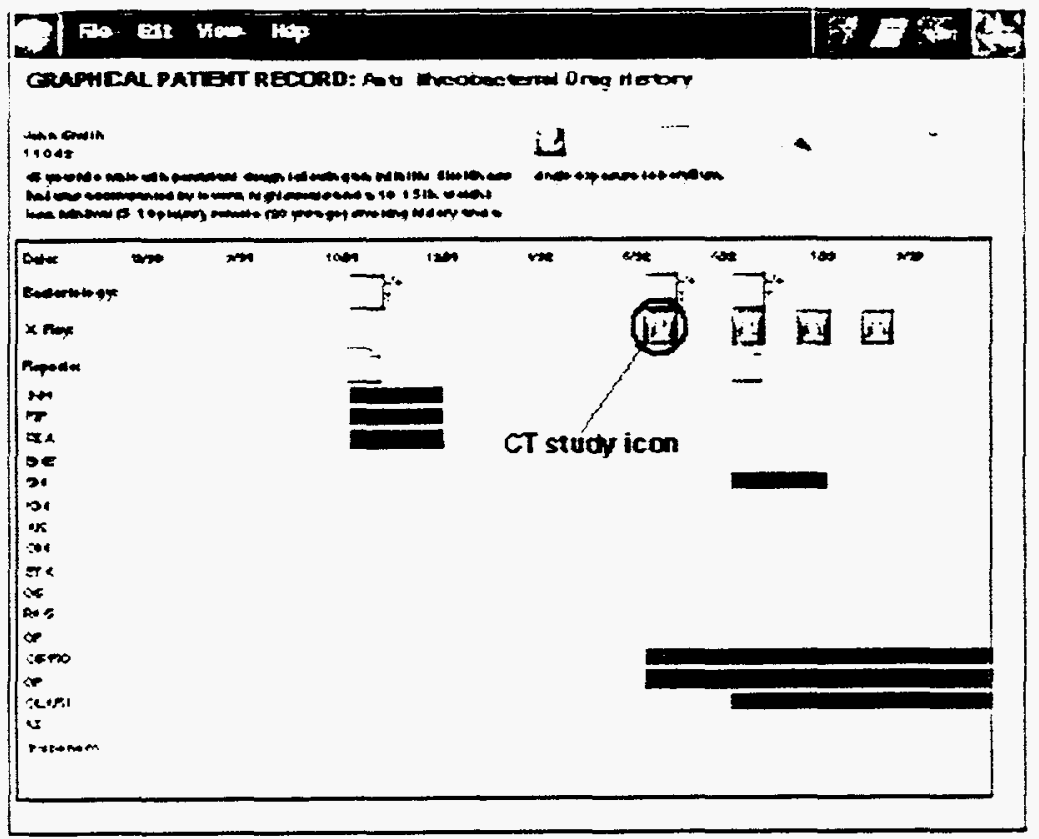

Figure 3. TeleMed Graphical Patient Record (GPR).

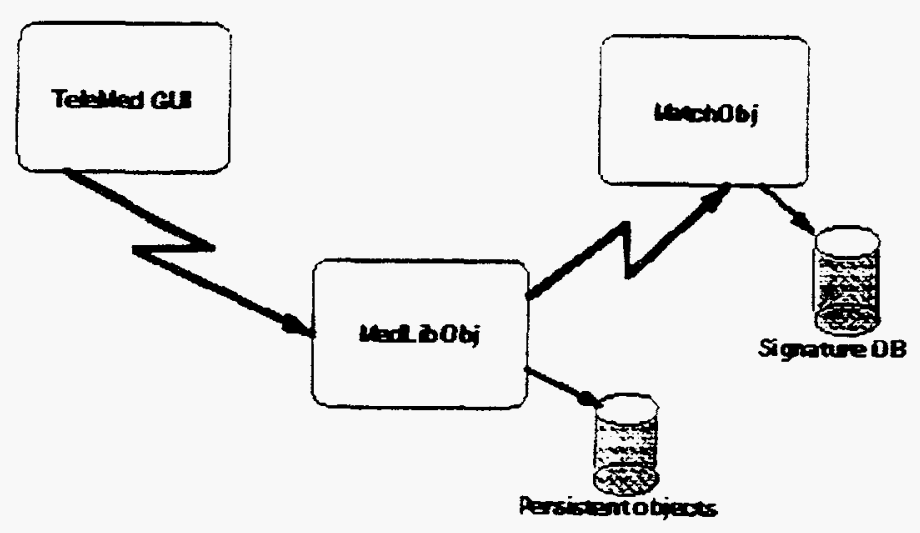

Figure 4. TeleMed object distribution. 


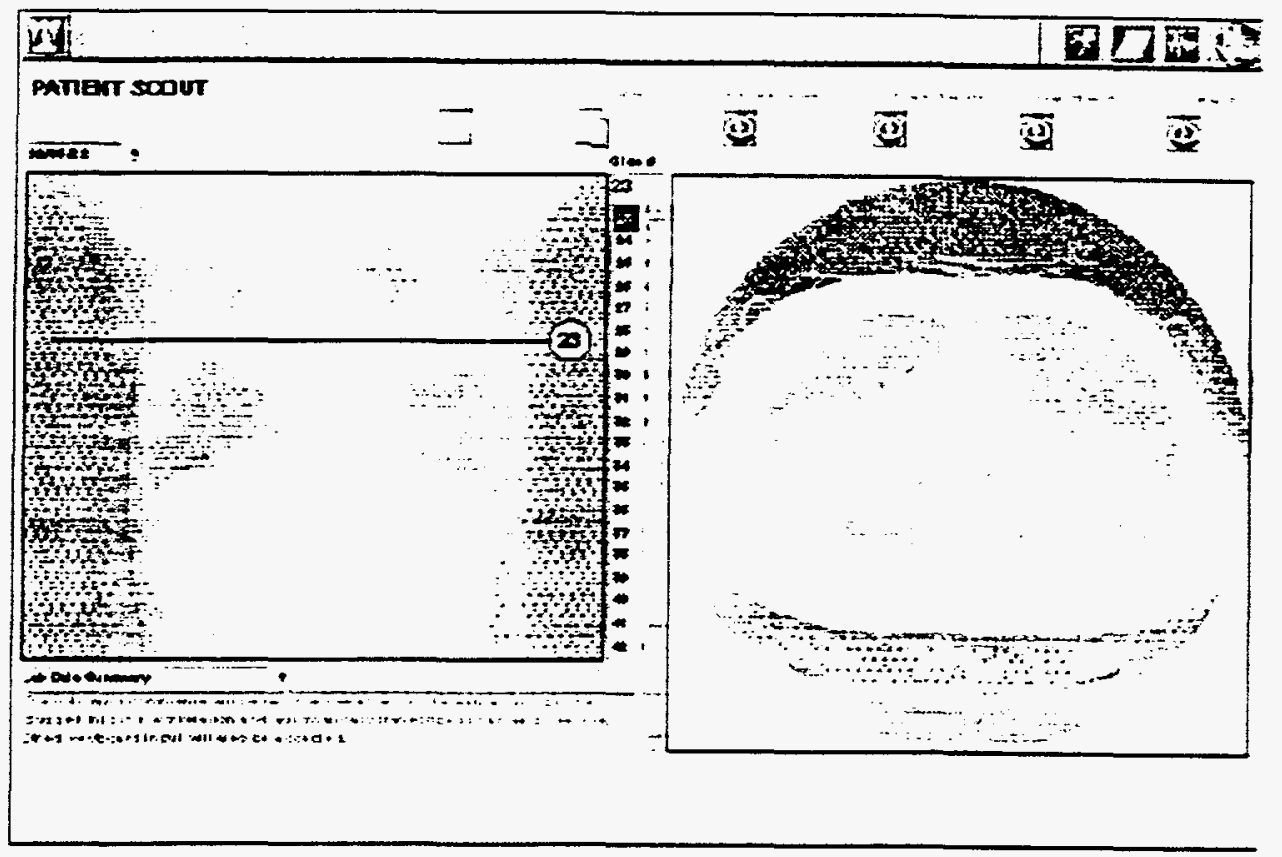

Figure 5. TeleMed CT study interface.

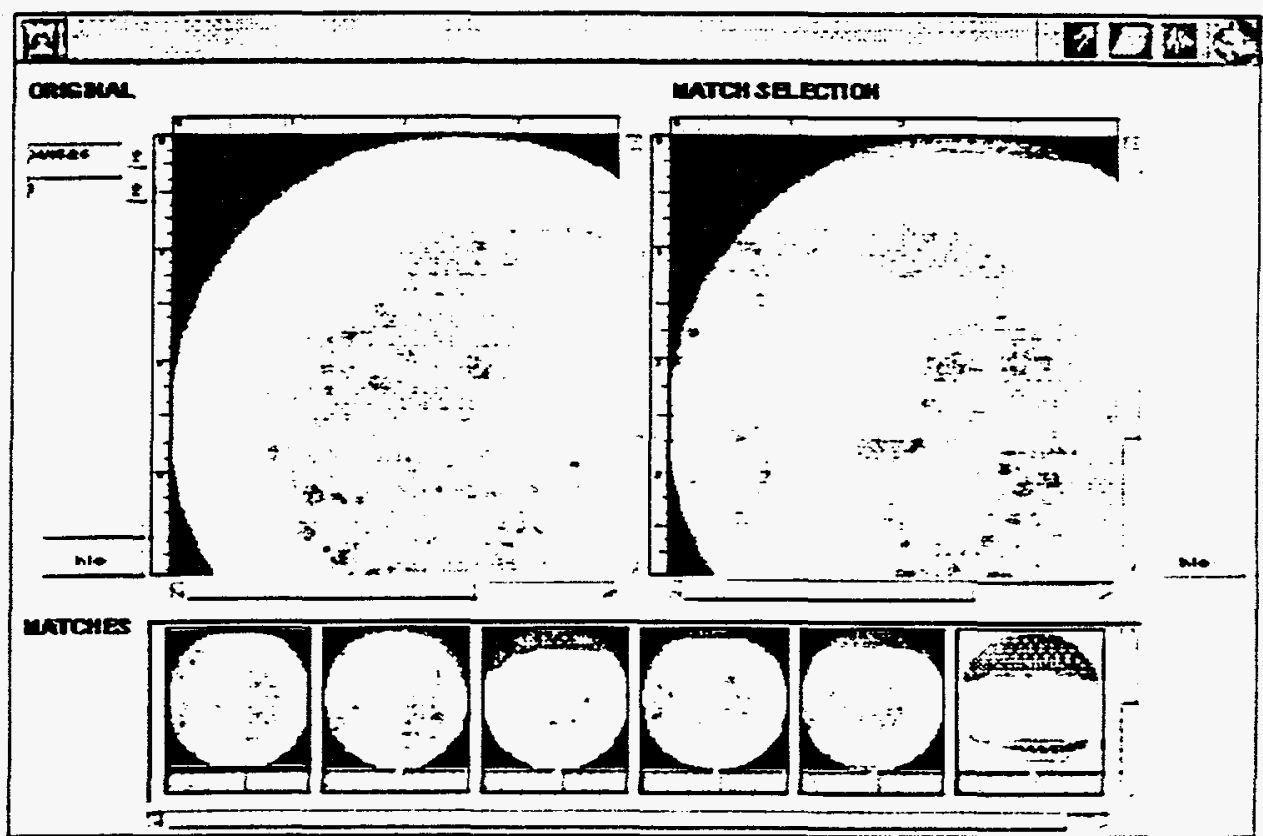

Figure 6. TeleMed image matching interface. 